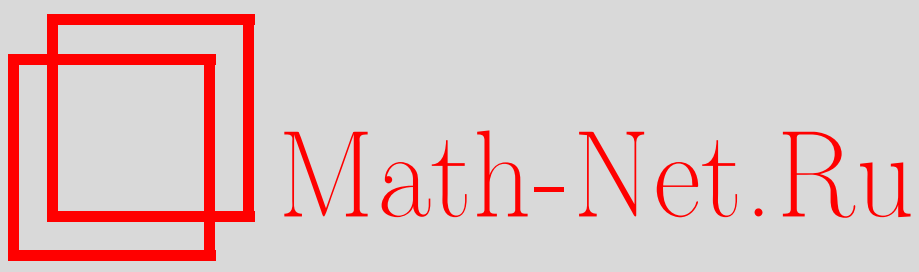

Б. П. Андреянов, О пределах решений задачи Римана для системы изэнтропической газовой динамики с вязкостью в эйлеровых координатах, Матем. сб., 2003, том 194, номер 6, 3-22

DOI: https://doi.org/10.4213/sm739

Использование Общероссийского математического портала Math-Net.Ru подразумевает, что вы прочитали и согласны с пользовательским соглашением

http: //www.mathnet.ru/rus/agreement

Параметры загрузки:

IP : 54.205 .225 .156

26 апреля 2023 г., 08:59:18 


\section{Б.П. Андреянов}

\section{О пределах решений задачи Римана для системы изэнтропической газовой динамики с вязкостью в эйлеровых координатах}

\footnotetext{
Для задачи $\rho_{t}+(\rho u)_{x}=0,(\rho u)_{t}+\left(\rho u^{2}+p(\rho)\right)_{x}=0,\left.(\rho, u)\right|_{t=0, x<0}=$ $\left(\rho_{-}, u_{-}\right),\left.(\rho, u)\right|_{t=0, x>0}=\left(\rho_{+}, u_{+}\right)$доказьвается существование и единственность решения, которое может быть получено как предел при стремлении $\varepsilon>0$ к нулю ограниченного автомодельного решения регуляризованной задачи с дополнительньм членом $\varepsilon t u_{x x}$ во втором уравнении. Подробно описывается структура решений, в частности, в случае решений с вакуумом.

Библиограф̆ия: 19 названий.
}

\section{Введение}

Рассмотрим задачу

$$
\left\{\begin{array}{l}
\rho_{t}+(\rho u)_{x}=0 \\
(\rho u)_{t}+\left(\rho u^{2}+p(\rho)\right)_{x}=\varepsilon t u_{x x}
\end{array}\right.
$$

с начальным условием

$$
\rho(0, x)=\left\{\begin{array}{ll}
\rho_{+}, & x>0, \\
\rho_{-}, & x<0,
\end{array} \quad u(0, x)= \begin{cases}u_{+}, & x>0 \\
u_{-}, & x<0\end{cases}\right.
$$

где $\rho_{+}, \rho_{-} \in \mathbb{R}^{+}$и $u_{+}, u_{-} \in \mathbb{R}$. В настоящей статье изучаются ограниченные автомодельные (зависящие только от переменной $x / t$ ) решения задачи $(1),(2)$ и их сходимость при $\varepsilon \downarrow 0$.

В модели изэнтропической газовой динамики в эйлеровых координатах решения $(\rho, u):(t, x) \in \mathbb{R}^{+} \times \mathbb{R} \mapsto(\rho(t, x), u(t, x)) \in \mathbb{R}^{+} \times \mathbb{R}$ представляют собой плотность и скорость газа, $p$ - давление, а $\varepsilon>0$ - коэффициент вязкости. В дальнейшем предполагается, что $\rho_{ \pm}>0$, а $p(\cdot)$ является непрерывной и строго возрастаюшей на $\mathbb{R}^{+}$функцией с $p(0)=0$. Решения задачи $(1),(2)$ могут содержать точки с нулевой плотностью $\rho$; в дальнейшем мы будем называть такие решения "решениями с вакуумом".

Задача (1), (2) изучалась в работе [1] при помощи метода, использованного в [2] (см. также [3]). При дополнительных ограничениях, запрещающих появление вакуума в решениях, было доказано сушествование решений задачи (1), (2). Была получена равномерная по $\varepsilon>0$ оценка вариации решений и описана структура “веера волн” в пределе при $\varepsilon \downarrow 0$. 
В настоящей статье для широкого класса функций $p(\cdot)$ дается описание решений (1), (2), в которых допускается наличие вакуума.

В $\S 3$ рассматривается система

$$
\left\{\begin{array}{l}
\rho_{t}+(\rho u)_{x}=0, \\
(\rho u)_{t}+\left(\rho u^{2}+p(\rho)\right)_{x}=0
\end{array}\right.
$$

и при помощи определенных ниже (см. определение 1) оболочек функции $p(\cdot)$ дается формула для единственного решения задачи Римана (3), (2), которое может быть получено как предел ограниченных автомодельных решений задачи (1), (2) с $\varepsilon=\varepsilon_{n}$ для последовательности $\varepsilon_{n} \downarrow 0$.

Метод автомодельной исчезающей вязкости был использован в [4]-[10] для определения допустимости слабых решений задачи Римана для гиперболических систем законов сохранения. Следуя [11], назовем этот критерий допустимости критерием веера волн. Он был применен к исследованию различных специальных систем и различных вязкостных регуляризаций; см. обзор результатов в [12], [3]. В частности, задача (3), (2) с добавленной автомодельной вязкостью в форме $\varepsilon t\left(\begin{array}{l}\rho \\ \rho u\end{array}\right)$ изучалась в [13], в том числе и в случае решений с вакуумом. В работе [14] особое внимание было уделено образованию вакуума для этой задачи. В [3] существование решения, допустимого согласно критерию веера волн, было доказано для широкого класса строго гиперболических систем при условии малости разрыва в случае единичной матрицы вязкости.

Основным результатом настоящей статьи (теорема 2) является существование и единственность допустимого по критерию веера волн (определение 3) слабого решения задачи Римана для нестрого гиперболической системы (3) в случае матрицы вязкости $\left(\begin{array}{ll}0 & 0 \\ 0 & 1\end{array}\right)$ при дополнительном допущении, что

$$
p(\rho) \rightarrow+\infty \text { при } \rho \rightarrow+\infty .
$$

Структура этого решения описывается в $\S 3$.

Введем некоторые обозначения и определения, используемые в статье. Для заданных $a, b \in\{-\infty\} \cup \mathbb{R} \cup\{+\infty\}$ будем обозначать интервал $(\min \{a, b\}, \max \{a, b\})$ через $I(a, b)$, и через $\overline{I(a, b)}$ - его замыкание.

Выпуклой вниз (соответственно вверх) оболочкой функции $f(\cdot)$ на отрезке $\bar{I} \subset \mathbb{R}$ назовем наибольшую (соответственно наименьшую) выпуклую вниз (соответственно вверх) функцию $F(\cdot)$ такую, что $F \leqslant f$ на $\bar{I}$ (соответственно $F \geqslant f$ на $\bar{I})$.

Введем преобразование

$$
T:[F: V \in(0,+\infty) \mapsto F(V)] \mapsto[P: \rho \in(0,+\infty) \mapsto-F(1 / \rho)]
$$

и обозначим через $T^{-1}$ преобразование, обратное к $T$. В модели газовой динамики преобразование $T$ соответствует переходу от эйлеровых к лагранжевым координатам. Задача в лагранжевых координатах, эквивалентная задаче (1), (2) для решений без вакуума, была подробно изучена в [2] и [15] (результаты о допустимости решений для задачи без вязкости в лагранжевых координатах были получены в [16], [17]). Настоящая статья опирается, главньм образом, на анализ, проведенньй в [15]. 
ОПредЕЛЕниЕ 1 . Пусть $p: \mathbb{R}^{+} \rightarrow \mathbb{R}$ - непрерывная строго возрастаюшая функция, $a \geqslant 0$ и $b \geqslant a$. Нижней оболочкой функции $p(\cdot)$ на $[a, b]$ назовем функцию $P(\cdot)$, удовлетворяющую условиям:

(i) $P(\cdot) \in C[a, b]$ и $P \leqslant p$ на $[a, b]$;

(ii) функция $F=T^{-1} P$ выпукла вверх на $(1 / b, 1 / a)$;

(iii) для любой функции $Q(\cdot)$, удовлетворяюшей (i) и (ii), $P \geqslant Q$ на $[a, b]$.

Для $b>0$ и $a \geqslant b$, назовем верхней оболочкой $p(\cdot)$ на $[b, a]$ функцию $P(\cdot)$ такую, что:

$\left(\mathrm{i}^{\prime}\right) P(\cdot) \in C[b, a]$ и $P \geqslant p$ на $[b, a]$;

(ii') функция $F=T^{-1} P$ выпукла вниз на $(1 / a, 1 / b)$;

$\left(\mathrm{iii}^{\prime}\right)$ для любой функции $Q(\cdot)$, удовлетворяющей $\left(\mathrm{i}^{\prime}\right)$ и $\left(\mathrm{ii}^{\prime}\right), P \leqslant Q$ на $[b, a]$.

Назовем оболочкой $p(\cdot)$ на $\overline{I(a, b)}$ нижнюю оболочку на $[a, b]$ в случае $a \leqslant b$ и верхнюю оболочку на $[b, a]$ в случае $a>b$.

Для $a=0$ формально положим в (6) $1 / a=+\infty$. Отметим, что для любых $a, b \in \mathbb{R}^{+}$оболочка $P(\cdot)$ функции $p(\cdot)$ на $\overline{I(a, b)}$ однозначно определена. Для $a, b \neq 0$ имеем $P=T F$, где $F(\cdot)$ является вьпуклой вверх (соответственно вниз) оболочкой функции $f=T^{-1} p$ на $[1 / b, 1 / a]$ (соответственно на $[1 / a, 1 / b]$ ). Для $a=0 P(\cdot)$ строится как предел убывающей последовательности функций $P_{\delta}(\cdot)$ такой, что $\left.P_{\delta}(\cdot)\right|_{[0, \delta]} \equiv p(\delta)$ и $\left.P_{\delta}(\cdot)\right|_{[\delta, b]}$ удовлетворяет (6) на отрезке $[\delta, b]$; свойства $(6)$ легко следуют из монотонности и непрерывности $P(\cdot)$.

\section{§1. Некоторые свойства решений задачи с вязкостью}

Фиксируем $\varepsilon>0$.

ОПРеДЕЛЕНИЕ 2 . Пара функций $(\rho, u): \mathbb{R}^{+} \times \mathbb{R} \rightarrow \mathbb{R}^{+} \times \mathbb{R}$ называется решением задачи Римана (1), (2), если для любого $k>0(\rho, u)(t, x)=(\rho, u)(k t, k x)$ для п.в. $(t, x) \in \mathbb{R}^{+} \times \mathbb{R}, \rho, u \in L^{\infty}\left(\mathbb{R}^{+} \times \mathbb{R}\right)$, уравнения (1) выполнены в пространстве распределений $\mathscr{D}^{\prime}\left(\mathbb{R}^{+} \times \mathbb{R}\right)$ и

$$
\underset{t \downarrow 0}{\operatorname{ess}} \lim \left(\|\rho(t, \cdot)-\rho(0, \cdot)\|_{L^{1}(-R, R)}+\|u(t, \cdot)-u(0, \cdot)\|_{L^{1}(-R, R)}\right)=0
$$

для любого $R>0$, где $\rho(0, \cdot), u(0, \cdot)$ заданы формулой $(2)$.

Будем обозначать $x / t$ через $\xi$; в дальнейшем не делается различия между автомодельной функцией переменных $(t, x)$ и соответствуюшей функцией $\xi$.

ЛЕмма 1. Пара функиий $(\rho, u)$ является решением задачи (1), (2) в смысле определения 2 тогда и только тогда, когда выполнены следующие условия:

(i) существуют непрерывные ограниченные функции $\rho, u: \mathbb{R} \rightarrow \mathbb{R}$ такие, что $u^{\prime}(\cdot)$ u $(\cdot-u(\cdot)) \rho^{\prime}(\cdot)$ являются непрерывными $u(\rho, u)(t, x)=$ $(\rho, u)(x / t)$ для п.в. $(t, x) \in(0,+\infty) \times \mathbb{R}$;

(ii) существует постоянная $C \in \mathbb{R}$ такая, что

$$
\begin{gathered}
\varepsilon u^{\prime}(\xi)=-\int_{0}^{\xi}(\zeta-u(\zeta))^{2} \rho^{\prime}(\zeta) d \zeta+p(\rho(\xi))+C, \\
\rho(\xi) u^{\prime}(\xi)=(\xi-u(\xi)) \rho^{\prime}(\xi), \\
\lim _{\xi \rightarrow \pm \infty} \rho(\xi)=\rho_{ \pm}, \quad \lim _{\xi \rightarrow \pm \infty} u(\xi)=u_{ \pm} .
\end{gathered}
$$


Кроме того, существует единственное значение $\xi_{0}$ такое, что $и\left(\xi_{0}\right)=\xi_{0}$.

В случае $\rho\left(\xi_{0}\right)>0$ существуют $\xi_{ \pm} \in \overline{\mathbb{R}}$ такие, ито $\xi_{-} \leqslant \xi_{0} \leqslant \xi_{+}$и обе функиии $\rho(\cdot), u(\cdot)$ постоянны на $\left(\xi_{-}, \xi_{+}\right)$и строго монотонны на промежутках $\left(-\infty, \xi_{-}\right) u\left(\xi_{+},+\infty\right)$.

В случае $\rho\left(\xi_{0}\right)=0$ $\xi_{0}$ является единственной точкой вакуума в решении, $\xi_{ \pm}=\xi_{0}$ и имеют место аналогичнье свойства монотонности.

Более того, $u^{\prime}(\xi) \neq 0$ для всех $\xi \in\left(-\infty, \xi_{-}\right) \cup\left(\xi_{+},+\infty\right), u^{\prime}\left(\xi_{0}\right)=0$ в случае $\rho\left(\xi_{0}\right)>0$ u $0 \leqslant u^{\prime}\left(\xi_{0}\right)<1$ в случае $\rho\left(\xi_{0}\right)=0$.

ЗАмечАниЕ 1 . Из леммы 1 следует, что при $\varepsilon>0$ решения (1), (2) содержат не более одной точки вакуума. Отметим, что результат об отсутствии в решениях промежутков с нулевой плотностью для системы (1) с более традиционной вязкостью $\varepsilon u_{x x}$ во втором уравнении доказан в [18] для решений задачи Коши с любыми начальными данными.

ДоКАЗАТЕЛЬСТво проводится в четыре этапа.

I) Пусть $(\rho, u)$ является решением $(1),(2)$. Тогда $(\rho, u)(t, x)=(\rho, u)(x / t)$ и в $\mathscr{D}^{\prime}(\mathbb{R})$ имеют место равенства

$$
\left\{\begin{array}{l}
-\xi \rho^{\prime}+(\rho u)^{\prime}=0 \\
-\xi(\rho u)^{\prime}+\left(\rho u^{2}+p(\rho)\right)^{\prime}=\varepsilon u^{\prime \prime}
\end{array}\right.
$$

Из (12) вытекает, что $\varepsilon u^{\prime \prime}=-\xi^{2} \rho^{\prime}+\left(\rho u^{2}+p(\rho)\right)^{\prime}=-\left(\xi^{2} \rho\right)^{\prime}+2 \xi \rho+\left(\rho u^{2}+p(\rho)\right)^{\prime}$ в $\mathscr{D}^{\prime}(\mathbb{R})$, откуда $u^{\prime} \in L_{\text {loc }}^{\infty}(\mathbb{R})$ и $u \in C(\mathbb{R})$. Таким образом, $\rho u^{\prime},(\rho u) u^{\prime}$ имеют смысл в $\mathscr{D}^{\prime}(\mathbb{R})$, следовательно, $\rho^{\prime} u, \rho^{\prime} u^{2}$ тоже. Отсюда вытекает, что

$$
-(\xi-u) \rho^{\prime}+\rho u^{\prime}=0 \quad \text { в } \quad \mathscr{D}^{\prime}(\mathbb{R})
$$

и что

$$
-(\xi-u)^{2} \rho^{\prime}+p(\rho)^{\prime}=\varepsilon u^{\prime \prime} \quad \text { в } \quad \mathscr{D}^{\prime}(\mathbb{R}) .
$$

Следовательно, $(\xi-u) \rho=\int_{0}^{\xi} \rho(\zeta) d \zeta+$ const $\in C(\mathbb{R})$. Отсюда получаем, что $(\xi-u)^{2} \rho+p(\rho)-\varepsilon u^{\prime}=\int_{0}^{\xi} 2(\zeta-u(\zeta)) \rho(\zeta) d \zeta+$ const $\in C^{1}(\mathbb{R})$. Тогда

$$
p(\rho(\cdot))-\varepsilon u^{\prime}(\cdot) \in C(\mathbb{R}) .
$$

II) Теперь рассмотрим функцию $\xi \mapsto \xi-u(\xi)$. Она непрерывна и стремится к $\pm \infty$ при $\xi \rightarrow \pm \infty$. Поэтому существуют конечные значения $\eta_{-}:=\min \{\xi:$ $u(\xi)=\xi\}$ и $\eta_{+}:=\max \{\xi: u(\xi)=\xi\}$. При этом $\xi-u(\xi)<0$ на $\left(-\infty, \eta_{-}\right)$и $\xi-u(\xi)>0$ на $\left(\eta_{+},+\infty\right)$. Согласно (13) на каждом из этих промежутков имеем $\rho^{\prime} \in L_{\mathrm{loc}}^{\infty}$. Поэтому $\rho$ непрерьвна на этих промежутках; $u^{\prime}$ тоже - согласно (15); $\rho^{\prime}$ тоже - согласно (13).

Докажем, что $\rho, u$ монотонны на $\left(-\infty, \eta_{-}\right)$и на $\left(\eta_{+},+\infty\right)$. Возьмем $\xi_{+}:=\sup \left\{\xi \geqslant \eta_{+}:\left.\rho\right|_{\left(\eta_{+}, \xi\right]} \equiv\right.$ const $\}$. Сперва покажем, что $u$ строго монотонна на $\left(\xi_{+},+\infty\right)$. Действительно, предположим противное. Тогда существуют $c \in\left(\xi_{+},+\infty\right)$ и $0<\delta<c-\xi_{+}$такие, что $u^{\prime}(c)=0$ и $u^{\prime} \neq 0$ на $(c-\delta, c)$. 
Предположим для определенности, что $u^{\prime}>0$ на $(c-\delta, c)$. Из (13) получаем, что $\rho^{\prime}=\frac{\rho u^{\prime}}{\xi-u} \geqslant 0$ на $(c-\delta, c)$; следовательно, $p(\rho(\cdot))$ не убывает на $[c-\delta, c]$. Из (13), (14) получаем, что

$$
\varepsilon u^{\prime}(\xi)=\int_{\xi}^{c}(\zeta-u) \rho u^{\prime} d \zeta+p(\rho(\xi))-p(\rho(c)) \leqslant \int_{\xi}^{c}(\zeta-u) \rho u^{\prime} d \zeta
$$

поточечно на отрезке $[c-\delta, c]$. Выбирая последовательность $\xi_{n} \uparrow c$ такую, что $u^{\prime}\left(\xi_{n}\right)=\max _{\xi \in\left[\xi_{n}, c\right]} u^{\prime}(\xi)$, находим, что $\varepsilon u^{\prime}\left(\xi_{n}\right) \leqslant u^{\prime}\left(\xi_{n}\right) \mathscr{O}\left(c-\xi_{n}\right)$, где $\mathscr{O}(\cdot)$ обозначает некоторую ограниченную функцию. При $n \rightarrow \infty$ находим $\varepsilon \leqslant 0$, что противоречит условию.

Таким образом, приходим к заключению, что $u$ строго монотонна на $\left(\xi_{+},+\infty\right)$; согласно (13) и определению $\xi_{+} \rho$ строго монотонна на $\left(\xi_{+},+\infty\right)$. Аналогично, существует $\xi_{-} \leqslant \eta_{-}$такое, что $u, \rho$ строго монотонны на $\left(-\infty, \xi_{-}\right)$и $\rho \equiv$ const на $\left[\xi_{-}, \eta_{-}\right)$. Далее, на $\left(\eta_{+}, \xi_{+}\right]$и $\left[\xi_{-}, \eta_{-}\right)$имеем $\rho \equiv$ const; согласно (14) $u^{\prime}$ постоянна на каждом из этих промежутков. Учитывая непрерывность $u^{\prime}$ в точках $\xi_{ \pm}$, заключаем, что $u$ монотонна на $\left(-\infty, \eta_{-}\right)$и $\left(\eta_{+},+\infty\right)$.

III) Изучим поведение $\rho, u$ на промежутке $\left[\eta_{-}, \eta_{+}\right]$. Сперва отметим, что существуют конечные пределы $\rho\left(\eta_{ \pm} \pm 0\right)$; согласно (15) существуют конечные пределы $u^{\prime}\left(\eta_{ \pm} \pm 0\right)$.

Отметим, что $u^{\prime}\left(\eta_{+}+0\right)=0$ в случае $\rho\left(\eta_{+}+0\right)>0$ и $0 \leqslant u^{\prime}\left(\eta_{+}+0\right)<1$ в случае $\rho\left(\eta_{+}+0\right)=0$. Такая же связь имеет место между $u^{\prime}\left(\eta_{-}-0\right)$ и $\rho\left(\eta_{-}-0\right)$.

Действительно, положим $\rho\left(\eta_{+}+0\right)>0$. Из (13) получаем, что

$$
\int_{\eta_{+}}^{\eta_{+}+1}(\ln \rho(\zeta))^{\prime} d \zeta=\int_{\eta_{+}}^{\eta_{+}+1} \frac{u^{\prime}(\zeta)}{\zeta-u(\zeta)} d \zeta
$$

Интеграл в левой части сходится, поэтому предел $u^{\prime}(\eta+0)$ должен быть равен нулю. Далее, пусть $\rho\left(\eta_{+}+0\right)=0$. Тогда $\rho$ не убывает на $\left(\eta_{+},+\infty\right)$, откуда $u^{\prime}\left(\eta_{+}+0\right) \geqslant 0$ согласно (13). С другой стороны, из определения $\eta_{+}$немедленно вытекает, что $u^{\prime}\left(\eta_{+}+0\right) \leqslant 1$. Предположим, что $u^{\prime}\left(\eta_{+}+0\right)=1$. Из (14), (13) получаем

$$
\varepsilon\left(1-u^{\prime}(\xi)\right)=\int_{\eta_{+}}^{\xi}(\zeta-u(\zeta)) \rho(\zeta) u^{\prime}(\zeta) d \zeta-p(\rho(\xi))
$$

для всех $\xi \in\left(\eta_{+},+\infty\right)$. Так как $\rho u^{\prime}$ непрерывна на этом промежутке и стремится к нулю при $\xi \downarrow \eta_{+}$, сушествует $\delta>0$ такое, что

$$
\varepsilon(\xi-u(\xi))^{\prime} \leqslant \int_{\eta_{+}}^{\xi}(\zeta-u(\zeta)) d \zeta
$$

для всех $\xi \in\left(\eta_{+}, \eta_{+}+\delta\right)$. Полагая $g(\xi):=\xi-u(\xi)$ и $h(\xi):=\sqrt{\varepsilon} g(\xi)+\int_{\eta_{+}}^{\xi} g(\zeta) d \zeta$, из (16) получаем, что $h \in C^{1}\left[\eta_{-},+\infty\right)$ и $h^{\prime}(\xi) \leqslant 1 / \sqrt{\varepsilon} h(\xi)$. Так как $h\left(\eta_{+}\right)=0$, по неравенству Гронуолла получаем, что $h \equiv 0$ на $\left[\eta_{+}, \eta_{+}+\delta\right]$. Это противоречит 
определению $\eta_{+}$. Окончательно находим $0 \leqslant u^{\prime}\left(\eta_{+}+0\right)<1$. Доказательство для $\eta_{-}-0$ вместо $\eta_{+}+0$ проводится аналогично.

Теперь рассмотрим отдельно три случая.

a) $\eta_{-}=\eta_{+}=: \xi_{0}$ и один из пределов $\rho\left(\xi_{0} \pm 0\right)$ отличен от нуля. Тогда эти два предела совпадают. Действительно, пусть $\rho\left(\xi_{0}+0\right)>0$. Сперва предположим, что $\rho\left(\xi_{0}-0\right)>0$; в этом случае $u^{\prime}\left(\xi_{0} \pm 0\right)=0$, так что $\rho\left(\xi_{0} \pm 0\right)$ совпадают согласно (15). Далее, пусть $\rho\left(\xi_{0}-0\right)=0$. В этом случае $p\left(\rho\left(\xi_{0}+0\right)\right)-\varepsilon u^{\prime}\left(\xi_{0}+0\right)=p\left(\rho\left(\xi_{0}+0\right)\right)>0$ и $p\left(\rho\left(\xi_{0}-0\right)\right)-\varepsilon u^{\prime}\left(\xi_{0}-0\right)=-\varepsilon u^{\prime}\left(\xi_{0}-0\right) \leqslant 0$, что противоречит (15). Таким образом, находим, что функции $\rho, u^{\prime}$ непрерывны на $\mathbb{R}$. Поэтому $(13),(14)$ могут быть переписаны в виде (9), (10).

Как видим, в этом случае существует единственное $\xi_{0}$ такое, что $u\left(\xi_{0}\right)=\xi_{0}$, при этом $\rho\left(\xi_{0}\right)>0$ и $u^{\prime}\left(\xi_{0}\right)=0$. Более того, учитывая определение $\xi_{ \pm}$, находим, что $\rho, u$ постоянны на $\left[\xi_{-}, \xi_{+}\right]$и что $u^{\prime}$, а значит, и $\rho^{\prime}$ отличны от нуля на $\left(-\infty, \xi_{-}\right) \cup$ $\left(\xi_{+},+\infty\right)$.

b) $\eta_{-}=\eta_{+}=: \xi_{0}$ и $\rho\left(\xi_{0} \pm 0\right)=0$. Тогда $\rho, u^{\prime}$ непрерывны на $\mathbb{R}$, откуда вытекают (9), (10). Кроме того, $\xi=\xi_{0}$ является единственной точкой вакуума. Действительно, положим $\xi_{+}=\sup \{\xi: \rho(\xi)=0\}$. В соотношении

$$
\int_{\xi_{+}}^{\xi_{+}+1}(\ln \rho(\zeta))^{\prime} d \zeta=\int_{\xi_{+}}^{\xi_{+}+1} \frac{u^{\prime}(\zeta)}{\zeta-u(\zeta)} d \zeta
$$

интеграл слева расходится. Следовательно, $\zeta-u(\zeta) \rightarrow 0$ при $\xi \downarrow \xi_{+}$, откуда $\xi_{+}=\xi_{0}$.

Таким образом, в этом случае существует единственное $\xi_{0}$ такое, что $u\left(\xi_{0}\right)=\xi_{0}$, при этом $\rho\left(\xi_{0}\right)=0$ и $0 \leqslant u^{\prime}\left(\xi_{0}\right)<1 ;$ кроме того, $u^{\prime}, \rho^{\prime}$ отличны от нуля на $\mathbb{R} \backslash\left\{\xi_{0}\right\}$.

c) $\eta_{-}<\eta_{+}$. Покажем, что на самом деле такое невозможно. Действительно, из $(13)$ получаем, что $\rho=((\xi-u) \rho)^{\prime}$ в $\mathscr{D}^{\prime}(\mathbb{R})$. Поэтому

$$
\int_{\eta_{-}}^{\eta_{+}} \rho(\zeta) d \zeta=\left.[(\xi-u(\xi)) \rho(\xi)]\right|_{\eta_{-}} ^{\eta_{+}}=0
$$

так что $\left.\rho\right|_{\left(\eta_{-}, \eta_{+}\right)} \equiv 0$. Из (14) получаем, что $\left.u^{\prime}\right|_{\left(\eta_{-}, \eta_{+}\right)} \equiv$ const; учитывая, что $u\left(\eta_{ \pm}\right)=\eta_{ \pm}$, находим, что $u^{\prime}\left(\eta_{ \pm} \mp 0\right)=1$. Кроме того, $0 \leqslant u^{\prime}\left(\eta_{ \pm} \pm 0\right)<1$ во всех случаях; рассуждая как в случае а), приходим к противоречию с (15).

Таким образом, заключаем, что $\rho(\cdot), u(\cdot)$ удовлетворяют $(9),(10)$ и имеют свойства непрерывности и монотонности, указанные в лемме 1. Из монотонности $\rho, u$ немедленно следует, что (11) вьполнено в том и только том случае, когда автомодельные функции $\rho(\cdot, \cdot), u(\cdot, \cdot)$ удовлетворяют (8).

IV) Напротив, из (9)-(11), учитывая непрерывность $\rho(\cdot), u(\cdot), u^{\prime}(\cdot)$ и $(\cdot-u(\cdot)) \rho^{\prime}(\cdot)$, находим, что $(\rho, u)(t, x):=(\rho, u)(x / t)$ является решением $(1),(2)$. Действительно, (12) очевидно. Кроме того, (9), (10) влечет монотонность $\rho(\cdot)$, $u(\cdot)$ на бесконечности. Таким образом, (8) также выполнено.

Используя результаты леммы 1 , положим $\rho_{0}:=\rho\left(\xi_{0}\right)$ и $k:=u^{\prime}\left(\xi_{0}\right)$; определим $\sigma:=\rho_{0}-k$. Отметим, что $\sigma \in(-1,+\infty)$ и

$$
\rho_{0}=(\sigma)^{+}=\max \{\sigma, 0\}, \quad k=(\sigma)^{-}=\max \{-\sigma, 0\} .
$$


Далее, разобьем $\mathbb{R}$ на три (быть может, пустых) промежутка $\left(-\infty, \xi_{-}\right),\left(\xi_{-}, \xi_{+}\right)$, $\left(\xi_{+}, \infty\right)$. Обратим функцию $\rho(\cdot)$ на $\left(-\infty, \xi_{-}\right)$и на $\left(\xi_{+},+\infty\right)$. Определим функции

$$
\rho_{-}^{-1}: I\left(\rho_{0}, \rho_{-}\right) \rightarrow\left(-\infty, \xi_{-}\right), \quad \rho_{+}^{-1}: I\left(\rho_{0}, \rho_{+}\right) \rightarrow\left(\xi_{+},+\infty\right)
$$

в дальнейшем будем обозначать одной и той же буквой функцию $\rho(\cdot)$ и $\rho \in \mathbb{R}^{+}$как независимую переменную. Положим

$$
\Pi_{ \pm}^{\varepsilon}(\rho ; \sigma):=\int_{(\sigma)^{+}}^{\rho}\left[\rho_{ \pm}^{-1}(r)-u\left(\rho_{ \pm}^{-1}(r)\right)\right]^{2} d r-C
$$

для $\rho \in I\left(\rho_{0}, \rho_{ \pm}\right)$, где $C=\varepsilon u^{\prime}\left(\xi_{0}\right)-p\left(\rho_{0}\right)$. Будем использовать упрощенное обозначение $\Pi_{ \pm}(\cdot)$ для $\Pi_{ \pm}^{\varepsilon}(\cdot ; \sigma)$, когда рассматриваются фиксированные $\varepsilon, \sigma ;$ будем обозначать через “ · ” дифференцирование по переменной $\rho$. Уравнение (9) переписывается в виде

$$
\varepsilon u^{\prime}(\xi)=p(\rho(\xi))-\Pi_{ \pm}(\rho(\xi)) .
$$

Так как $0 \notin I\left(\rho_{0}, \rho_{ \pm}\right)$и показано, что $u^{\prime}(\xi)$ отлична от нуля на $\left(-\infty, \xi_{-}\right) \cup\left(\xi_{+},+\infty\right)$, заключаем, что $\Pi_{ \pm} \in C^{2}\left(I\left(\rho_{0}, \rho_{ \pm}\right)\right)$и что вьполнено

$$
\rho \ddot{\Pi}_{ \pm}+2 \dot{\Pi}_{ \pm}=\frac{2 \varepsilon \dot{\Pi}_{ \pm}}{p-\Pi_{ \pm}}, \quad \dot{\Pi}_{ \pm}>0 \quad \text { и } \operatorname{sign}\left(p-\Pi_{ \pm}\right)=\operatorname{sign}\left(\rho_{ \pm}-\rho_{0}\right)
$$

на $I\left(\rho_{0}, \rho_{ \pm}\right)$. Далее, $\Pi_{ \pm}$может быть продолжена по непрерывности на $\overline{I\left(\rho_{0}, \rho_{ \pm}\right)}$. Согласно (19) и лемме $1 \Pi_{ \pm}(\cdot)$ допускают конечные пределы в точке $\rho_{0}$, и можно положить

$$
\Pi_{ \pm}\left(\rho_{0}\right)=p\left(\rho_{0}\right)-\varepsilon k,
$$

где $\rho_{0}, k$ определены соотношением (17). Кроме того,

$$
\Pi_{ \pm}\left(\rho_{ \pm}\right)=p\left(\rho_{ \pm}\right)
$$

Действительно, правая часть в (19) имеет конечные пределы при $\rho(\xi) \rightarrow \rho_{ \pm}$, так как в случае $\rho_{0}<\rho_{ \pm}$функции $\Pi_{ \pm}(\cdot)$ возрастающие и ограничены сверху, а в случае $\rho_{0}>\rho_{ \pm}$они вьпуклы вверх и ограничены снизу. Находим, что $u^{\prime}(\xi) \rightarrow 0$ при $\xi \rightarrow \pm \infty$, так как $u$ имеет конечные пределы на $\pm \infty$.

В дополнение, в случаях $\xi_{-}=-\infty$ (т.е. $\left.\rho_{0}=\rho_{-}\right)$и $/$или $\xi_{+}=+\infty\left(\right.$ т.е. $\left.\rho_{0}=\rho_{+}\right)$, просто определим $\Pi_{-}(\cdot)$ и/или $\Pi_{+}(\cdot)$ соотношением $(22)$.

Наконец, из (18) и (10) находим $u^{\prime}(\xi)= \pm \sqrt{\dot{\Pi}_{ \pm}(\rho)} \frac{\rho^{\prime}(\xi)}{\rho}$ для всех $\xi \in\left(-\infty, \xi_{-}\right)$ и всех $\xi \in\left(\xi_{+},+\infty\right)$ соответственно. Учитывая, что $u\left(\xi_{-}\right)=u\left(\xi_{+}\right)$, из (11) получаем, что

$$
u_{+}-u_{-}=\int_{\rho_{0}}^{\rho_{+}} \sqrt{\dot{\Pi}_{+}(r)} \frac{d r}{r}+\int_{\rho_{0}}^{\rho_{-}} \sqrt{\dot{\Pi}_{-}(r)} \frac{d r}{r}
$$

и интегралы в правой части (23) конечны.

Таким образом, доказан следующий результат. 
ПРЕДЛОЖЕНИЕ 1. Пусть $(\rho, u)$ является решением (1), (2) в смысле определения 2. Тогда существуют числа $\sigma \in(-1,+\infty), \rho_{0} \in[0,+\infty], k \in[0,1) u$ функиии $\Pi_{ \pm} \in C\left(\overline{I\left(\rho_{0}, \rho_{ \pm}\right)}\right) \cap C^{2}\left(I\left(\rho_{0}, \rho_{ \pm}\right)\right)$такие, что выполнены соотношения (17) $и$ (20)-(23).

Верно и обратное утверждение.

ПРЕДЛОЖЕНИЕ 2. Пусть $\sigma \in(-1,+\infty)$, a $\rho_{0}, k$ определены соотношениями (17). Пусть $\Pi_{ \pm} \in C\left(\overline{I\left(\rho_{0}, \rho_{ \pm}\right)}\right) \cap C^{2}\left(I\left(\rho_{0}, \rho_{ \pm}\right)\right)$удовлетворяют $(20)-(23)$. Тогда существует решение $(\rho, u)$ задачи $(1),(2)$ в смысле определения 2 , это решение дается формулами

$$
\begin{gathered}
\rho(t, x)=\rho(x / t)= \begin{cases}{\left[\Xi_{-}^{\varepsilon}\right]^{-1}(x / t),} & x / t<\xi_{-}, \\
{\left[\Xi_{+}^{\varepsilon}\right]^{-1}(x / t),} & \xi_{+}<x / t,\end{cases} \\
\equiv \begin{cases}{\left[\Xi_{-}^{\varepsilon}\right]^{-1}(x / t),} & x / t<\xi_{-}, \\
\rho_{0}, & \xi_{-}<x / t<\xi_{+}, \\
{\left[\Xi_{+}^{\varepsilon}\right]^{-1}(x / t),} & \xi_{+}<x / t,\end{cases} \\
u(t, x)=u(x / t)= \begin{cases}U_{-}^{\varepsilon} \circ\left[\Xi_{-}^{\varepsilon}\right]^{-1}(x / t), & x / t<\xi, \\
U_{-}^{\varepsilon}\left(\rho_{0}\right)=U_{+}^{\varepsilon}\left(\rho_{0}\right), & \xi_{-}<x / t<\xi_{+}, \\
U_{+}^{\varepsilon} \circ\left[\Xi_{+}^{\varepsilon}\right]^{-1}(x / t), & \xi_{+}<x / t,\end{cases}
\end{gathered}
$$

$2 \partial e$

$$
\begin{array}{lll}
U_{ \pm}^{\varepsilon}(\rho):=u_{ \pm} \mp \int_{\rho}^{\rho_{ \pm}} \sqrt{\dot{\Pi}_{ \pm}(r)} \frac{d r}{r} \quad \partial \Omega_{\text {g }} & \rho \in \overline{I\left(\rho_{0}, \rho_{ \pm}\right)}, \\
\Xi_{ \pm}^{\varepsilon}(\rho):=U_{ \pm}^{\varepsilon}(\rho) \pm \sqrt{\dot{\Pi}_{ \pm}(\rho)} \quad \partial \Omega_{\text {g }} & \rho \in I\left(\rho_{0}, \rho_{ \pm}\right),
\end{array}
$$

а $\xi_{ \pm}$определеньц формулами

$$
\begin{array}{llll}
\xi_{ \pm}:=\lim _{\rho \in I\left(\rho_{0}, \rho_{ \pm}\right), \rho \rightarrow \rho_{0}} \Xi_{ \pm}^{\varepsilon}(\rho) & \text { npu } & \rho_{0} \neq \rho_{ \pm}, \\
\xi_{-}:=-\infty \quad \text { u/uлu } \xi_{+}:=+\infty & \text { npu } & \rho_{0}=\rho_{-} u / u л u \rho_{0}=\rho_{+} .
\end{array}
$$

ДокАЗАТЕльство. Случаи $\rho_{0}=\rho_{+}, \rho_{0}=\rho_{-}$тривиальны; предположим, что $\rho_{0} \neq \rho_{ \pm}$. Определим $U_{ \pm}^{\varepsilon}, \Xi_{ \pm}^{\varepsilon}$ соотношениями $(26),(27)$. Отметим, что оба интеграла в (26) должны сходиться при $\rho \rightarrow \rho_{0}, \rho \in I\left(\rho_{0}, \rho_{ \pm}\right)$. Действительно, в случае $\rho_{0}=0$ оба интеграла положительны и, следовательно, конечны согласно (23). В случае $\rho_{0}>0$ функции $\dot{\Pi}_{ \pm}$ограничены при $\rho \rightarrow \rho_{0}$ в силу монотонности $\rho^{2} \dot{\Pi}_{ \pm}$, которая очевидна из (20). Поэтому определены значения $U_{ \pm}^{\varepsilon}\left(\rho_{0}\right)$; согласно $(23)$ они совпадают. Отметим также, что функции

$$
\dot{\Xi}_{ \pm}^{\varepsilon}(\rho)= \pm \frac{\rho \ddot{\Pi}_{ \pm}(\rho)+2 \dot{\Pi}_{ \pm}(\rho)}{2 \rho \sqrt{\dot{\Pi}_{ \pm}(\rho)}}= \pm \frac{\varepsilon \sqrt{\dot{\Pi}_{ \pm}(\rho)}}{\rho\left(p(\rho)-\Pi_{ \pm}(\rho)\right)}
$$


непрерывны и отличны от нуля на $I\left(\rho_{0}, \rho_{ \pm}\right)$, так что можно определить $\xi_{ \pm}$, а также (многозначные) функции $\left[\Xi_{ \pm}^{\varepsilon}\right]^{-1}: I\left(\xi_{ \pm}, \pm \infty\right) \rightarrow \mathbb{R}^{+}$. Из $(23),(26),(27)$ находим, чTO

$$
\xi_{+}-\xi_{-}=\lim _{\rho \in I\left(\rho_{0}, \rho_{-}\right), \rho \rightarrow \rho_{0}} \sqrt{\dot{\Pi}_{-}(\rho)}+\lim _{\rho \in I\left(\rho_{0}, \rho_{+}\right), \rho \rightarrow \rho_{0}} \sqrt{\dot{\Pi}_{+}(\rho)} \geqslant 0
$$

Ясно, что $\rho, u \in C(\mathbb{R}) \cap C^{1}\left(\mathbb{R} \backslash\left\{\xi_{-}, \xi_{+}\right\}\right) ;$кроме того, (24)-(27), (29) и (22) влекут $(11),(19)$ для всех $\xi \neq \xi_{ \pm}$. На самом деле, можно показать, что из $(22),(20)$ следует, что $\sqrt{\dot{\Pi}_{ \pm}(\rho)} \rightarrow+\infty$ при $\rho \rightarrow \rho_{ \pm}, \rho \in I\left(\rho_{0}, \rho_{ \pm}\right)$, так что $\Xi_{ \pm}^{\varepsilon}$ отображают $I\left(\rho_{0}, \rho_{ \pm}\right)$на $\left(\xi_{+},+\infty\right)$ и $\left(-\infty, \xi_{-}\right)$соответственно. Но даже если допустить, что $\Xi_{ \pm}^{\varepsilon}$ ограничены, из (22) следует (19) для всех $\xi$ вне области значений $\Xi_{ \pm}^{\varepsilon}$. Более того, из $(29),(19),(27)$ и $(24),(25)$ получаем для всех $\xi \neq \xi_{ \pm}$

$$
(\xi-u(\xi)) \rho^{\prime}(\xi)= \pm \frac{\rho\left(p(\rho(\xi))-\Pi_{ \pm}(\rho(\xi))\right)}{ \pm \varepsilon}=\rho(\xi) u^{\prime}(\xi)
$$

значит, (10) вьполнено для $\xi \neq \xi_{ \pm}$.

Теперь рассмотрим два возможных случая.

а) $\rho_{0}>0$. Тогда $u^{\prime}\left(\xi_{ \pm} \pm 0\right)=0$ согласно (19) и (21), а в случае $\xi_{-}<\xi_{+}$из (25) имеем $u^{\prime}\left(\xi_{ \pm} \mp 0\right)=0$. Таким образом, $u \in C^{1}(\mathbb{R})$ и согласно $(31)(\cdot-u(\cdot)) \rho^{\prime}(\cdot)$ непрерывна на $\mathbb{R}$. Следовательно, (9), (10) выполнены всюду; свойство (11) очевидно.

b) $\rho_{0}=0$. Тогда $\dot{\Pi}_{ \pm}(\rho) \rightarrow 0$ при $\rho \rightarrow \rho_{0}, \rho \in I\left(\rho_{0}, \rho_{ \pm}\right)$. Действительно, из $(20)$ имеем для $\rho \in I\left(\rho_{0}, \rho_{ \pm}\right)$

$$
\int_{\rho}^{\left(\rho_{0}+\rho_{+}\right) / 2}\left(\ln \dot{\Pi}_{ \pm}(r)\right) \cdot d r=\int_{\rho}^{\left(\rho_{0}+\rho_{+}\right) / 2} \frac{2}{r}\left(\frac{\varepsilon}{p(r)-\Pi_{ \pm}(r)}-1\right) d r
$$

Из $(21),(17)$ вытекает, что $\varepsilon /\left(p(r)-\Pi_{ \pm}(r)\right)-1 \rightarrow 1 / k-1>0$ при $\rho \rightarrow \rho_{0}$. Поэтому интеграл справа расходится; значит, $\ln \dot{\Pi}_{ \pm}(\rho) \rightarrow-\infty$ при $\rho \rightarrow \rho_{0}, \rho \in I\left(\rho_{0}, \rho_{ \pm}\right)$. Из (30) заключаем, что $\xi_{-}=\xi_{+}$; из (19) и (21) находим, что $u^{\prime}\left(\xi_{ \pm} \pm 0\right)=k$. Таким образом, в этом случае $u^{\prime}(\cdot)$ и $(\cdot-u(\cdot)) \rho^{\prime}(\cdot)$ тоже непрерывны на $\mathbb{R}$ и выполнены (9)-(11).

Согласно лемме $1(\rho, u)$ является решением $(1),(2)$.

\section{§ 2. Существование и единственность решения задачи с вязкостью}

В $\S 1$ задача (1), (2) была сведена к нахождению чисел $\sigma \in(-1,+\infty), \rho_{0}, k$ и пары функций $\Pi_{ \pm} \in C\left(\overline{I\left(\rho_{0}, \rho_{ \pm}\right)}\right) \cap C^{2}\left(I\left(\rho_{0}, \rho_{ \pm}\right)\right)$, удовлетворяющих (17) и (20)-(23). В этом параграфе доказьвается, что такие $\sigma, \rho_{0}, k, \Pi_{ \pm}(\cdot)$ существуют (и единственны), если $p(\cdot)$ удовлетворяет условию (4) (см. предложение 3 и леммы 4-6). Также доказываются предварительные результаты о сходимости решений (см. лемму 3 и предложение 4). 
Для начала зафиксируем $\sigma \in[-1,+\infty)$ и $b \in(0,+\infty)$. Положим $a:=(\sigma)^{+}$, $k:=(\sigma)^{-}$и рассмотрим задачу нахождения функции $\Pi \in C(\overline{I(a, b)}) \cap C^{2}(I(a, b))$, удовлетворяюшей соотношениям

$$
\begin{gathered}
\rho \ddot{\Pi}(\rho)+2 \dot{\Pi}(\rho)=\frac{2 \varepsilon \dot{\Pi}(\rho)}{p(\rho)-\Pi(\rho)}, \\
\dot{\Pi}(\rho)>0, \quad(b-a)(p(\rho)-\Pi(\rho))>0 \text { для всех } \rho \in I(a, b), \\
\Pi(a)=p(a)-\varepsilon k, \quad \Pi(b)=p(b) .
\end{gathered}
$$

ПРеДЛОЖЕНИЕ 3. Решение задачи (32) существует и единственно.

Пусть $\Pi^{\varepsilon}(\cdot)$ обозначает решение $(32)$, соответствуюшее данному $\varepsilon, \varepsilon>0$.

ПрЕДЛОЖЕНИЕ 4. При $\varepsilon \downarrow 0 \Pi^{\varepsilon}$ сходится равномерно на $\overline{I(a, b)} \kappa$ оболочке функиии $p(\cdot)$ (см. определение 1$)$.

Для доказательства этих утверждений сперва отметим, что уравнение в (32) удовлетворяет принципу максимума. Дадим формулировку для случая $a<b$.

ЛЕмма 2 (принцип максимума). Пусть П, $\in C[a, b] \cap C^{2}(a, b)$ и удовлетворяют для всех $\rho \in(a, b)$ уравнениям $\ddot{\Pi}(\rho)=G(\rho, \Pi(\rho), \dot{\Pi}(\rho))$ и $\ddot{\Upsilon}(\rho)=$ $H(\rho, \Upsilon(\rho), \dot{\Upsilon}(\rho))$ соответственно с некоторыми $G, H:(a, b) \times \mathbb{R} \times(0,+\infty) \rightarrow$ $(0,+\infty]$.

(a) Предположим, что $G(\rho, z, w)<H(\rho, \zeta, w)$ для всех $\rho \in(a, b)$ таких, что $\Pi(\rho)<\Upsilon(\rho)$, и для всех $z, \zeta, w \operatorname{ma\kappa ux,~что~} z<\zeta$. Тогда $\Pi \geqslant \Upsilon$ на $[a, b]$, если $\Pi(a) \geqslant \Upsilon(a) u \Pi(b) \geqslant \Upsilon(b)$.

(b) Предположсим, что функиия $G(\rho, z, w)$ совпадает с $H(\rho, z, w)$ и строго возрастает по переменной $z ;$ пусть $\Pi(a)=\Upsilon(a)$ или $(b)=\Upsilon(b)$. Тогда $\Pi-\Upsilon$ монотонна на $[a, b]$.

Доказательство стандартно.

Далее, отметим следующую лемму, главным образом используемую в 3 . Пусть преобразование $T$ определяется формулой (5); как и в определении 1 , полагаем $1 / a=+\infty$ в случае $a=0$.

Лемма 3. Пусть $[a, b] \subset \mathbb{R}^{+}$, и пусть $\left\{P^{\varepsilon}\right\}_{\varepsilon \geqslant 0} \subset C[a, b]-$ множество функиий таких, что $F^{\varepsilon}=T^{-1} P^{\varepsilon}$ выпукльи вверх на $(1 / b, 1 / a)$. Предположим, что для всех $\rho \in[a, b] \quad P^{\varepsilon}(\rho)$ сходится $\kappa P^{0}(\rho)$ при $\varepsilon \downarrow 0$. Тогда выполнены следующие утвержсдения.

(a) Сходимость равномерна на каждом отрезке $[c, d] \subset(a, b)$, и для каждого $\varepsilon P^{\varepsilon}$ абсолютно непрерывна на каждом отрезке $[c, d] \subset(a, b)$. Более того, если $P^{\varepsilon}$ возрастающие, то $\dot{P}^{\varepsilon}$ ограничены равномерно по є на любом отрезке $[c, d] \subset[a, b)$ таком, что $c>0$.

(b) Для любой последовательности $\varepsilon_{n} \downarrow 0 \dot{P}^{\varepsilon_{n}} \rightarrow \dot{P}^{0}$ n.в. на $(a, b) ;$ причем если $P^{\varepsilon_{n}}, P^{0} \in C^{1}(a, b)$, сходимость имеет место всюду на $(a, b)$.

(c) Предположим, что $P^{\varepsilon}$ возрастающие и

$$
\Xi_{ \pm}^{\varepsilon}(\rho):=\text { const } \mp \int_{\rho}^{b} \sqrt{\dot{P}^{\varepsilon}(r)} \frac{d r}{r} \pm \sqrt{\dot{P}^{\varepsilon}(\rho)} .
$$


Тогда $\Xi_{ \pm}^{\varepsilon}(\cdot)$ являются п.в. определенными монотонными функииями на $(a, b)$, так что $\left[\Xi_{ \pm}^{\varepsilon}\right]^{-1}$ определены как монотонные многозначные функиии.

(d) Если $P^{\varepsilon}$ возрастают, для любой последовательности $\varepsilon_{n} \downarrow 0$, для любого отрезка $[c, d] \subset[a, b]$ такого, ито $c>0$, имеем

$$
\int_{\rho}^{b} \sqrt{\dot{P}^{\varepsilon_{n}}(r)} \frac{d r}{r} \rightarrow \int_{\rho}^{b} \sqrt{\dot{P}^{0}(r)} \frac{d r}{r}
$$

равномерно по $\rho \in[c, d]$.

(е) $В$ обозначениях (с) для любой последовательности $\varepsilon_{n} \downarrow 0$ имеем $\left[\Xi_{+}^{\varepsilon_{n}}\right]^{-1}(\xi) \rightarrow\left[\Xi_{+}^{0}\right]^{-1}(\xi)$ для всех $\xi$ таких, что $\left[\Xi_{+}^{0}\right]^{-1}$ непрерывна в точке $\xi$. То жие самое выполнено с $\left[\Xi_{-}^{\varepsilon_{n}}\right]^{-1},\left[\Xi_{-}^{0}\right]^{-1}$ вместо $\left[\Xi_{+}^{\varepsilon_{n}}\right]^{-1}$, $\left[\Xi_{+}^{0}\right]^{-1}$.

Аналогичные свойства имеют место в случае, когда $P^{\varepsilon}$ определены на $[b, a] \subset$ $\mathbb{R}^{+} \backslash\{0\}$ и $F^{\varepsilon}=T^{-1} P^{\varepsilon}$ выпуккль вниз на $(1 / a, 1 / b)$.

ДокаЗАтЕльство. Так как $F^{\varepsilon}=T^{-1} P^{\varepsilon}$ вьпуклы вверх, они дифференпируемы п.в. Более того, сходимость $P^{\varepsilon}(\rho)$ к $P^{0}(\rho)$ влечет сходимость $F^{\varepsilon}(1 / \rho)$ к $F^{0}(1 / \rho)$ при $\varepsilon \downarrow 0$. Имеем $F^{\varepsilon} \rightarrow F^{0}$ равномерно на любом отрезке $[1 / d, 1 / c] \subset(1 / b, 1 / a)$ и $\frac{d}{d V} F^{\varepsilon_{n}} \rightarrow \frac{d}{d V} F^{0}$ п.в. на $(1 / b, 1 / a)$ при $\varepsilon \downarrow 0$. Так как $\dot{P}^{\varepsilon}(\rho)=\frac{1}{\rho^{2}} \frac{d}{d V} F^{\varepsilon}(V)$ в случае, когда сушествует $\frac{d}{d V} F^{\varepsilon}(V),(\mathrm{a})$ и (b) очевидны.

Более того, $\frac{d}{d V} F^{\varepsilon} \geqslant 0$ в случае (c). Заменяя $v=1 / r$ в интеграле в формуле (33), получаем

$$
\Xi_{ \pm}^{\varepsilon}(\rho)=\mathrm{const} \pm \int_{1 / b}^{1 / \rho}\left(\sqrt{\frac{d}{d V} F^{\varepsilon}\left(\frac{1}{\rho}\right)}-\sqrt{\frac{d}{d V} F^{\varepsilon}(v)}\right) d v \pm \frac{1}{b} \sqrt{\frac{d}{d V} F^{\varepsilon}\left(\frac{1}{\rho}\right)} .
$$

Эта функция монотонна, так как $\frac{d}{d V} F^{\varepsilon}$ монотонна; отсюда получаем (c). Свойство $(\mathrm{d})$ следует из непрерывности и сходимости $F^{\varepsilon}(\cdot)$ в точке $V=1 / b$. Действительно, имеем для $\rho>c>0$

$$
\left|\int_{\rho}^{b} \sqrt{\dot{P}^{\varepsilon_{n}}(r)} \frac{d r}{r}-\int_{\rho}^{b} \sqrt{\dot{P}^{0}(r)} \frac{d r}{r}\right| \leqslant \int_{1 / b}^{1 / \rho}\left|\sqrt{\frac{d}{d V} F^{\varepsilon_{n}}(v)}-\sqrt{\frac{d}{d V} F^{0}(v)}\right| d v
$$

Возьмем $\delta>0$ и проинтегрируем $\left|\sqrt{\frac{d}{d V} F^{\varepsilon} n(V)}-\sqrt{\frac{d}{d V} F^{0}(V)}\right|$ отдельно по интервалам $(1 / b, 1 / b+\delta)$ и $(1 / b+\delta, 1 / c)$. Для любого $\delta>0$ второй интеграл стремится к нулю при $\varepsilon_{n} \downarrow 0$ по свойству (а) и теореме Лебега об ограниченной сходимости. При этом первый интеграл может быть сделан сколь угодно малым при выборе 
достаточно малого $\delta$, потому что

$$
\begin{aligned}
\int_{1 / b+\delta}^{1 / b} \sqrt{\frac{d}{d V} F^{\varepsilon_{n}}(v)} d v & \leqslant \int_{1 / b+\delta}^{1 / b}\left(1+\frac{d}{d V} F^{\varepsilon_{n}}(v)\right) d v \\
& =\delta+\left(F^{\varepsilon_{n}}\left(\frac{1}{b}+\delta\right)-F^{\varepsilon_{n}}\left(\frac{1}{b}\right)\right) \\
& \leqslant 2 \delta+\left(F^{0}\left(\frac{1}{b}+\delta\right)-F^{0}\left(\frac{1}{b}\right)\right)
\end{aligned}
$$

для достаточно малых $\varepsilon_{n}$. Следовательно, левая часть (34) может быть сделана сколь угодно малой равномерно по $\rho \in[c, d]$. Более того, если $a>0$, можно положить $c=a$ в приведенном выше рассуждении и доказать $(\mathrm{d})$.

Наконец, из (b) и (d) следует, что $\Xi_{ \pm}^{\varepsilon_{n}} \rightarrow \Xi_{ \pm}^{0}$ п.в. на $(a, b)$. В элементарной теории вероятностей хорошо известно, что сходимость почти всюду последовательности монотонных функций (случайных величин) влечет поточечную сходимость их обратных функций (функций распределения) в точках непрерывности предельной функции. Отсюда заключаем (е).

Случай вьпуклых вниз функций $F^{\varepsilon}$ разбирается аналогично.

ДоКАЗАТЕЛЬСТво ПРЕДЛОЖЕНИЯ 3. Случай $a=b$ тривиален; для определенности положим $a<b$, что является самьм сложным случаем. Единственность немедленно следует из леммы 2 . Для любого $a>0$ сушествование решения можно доказать при помощи пенализации правой части (32), применяя леммы 2,3 по аналогии с [15]. Вместо этого выполним преобразование $T^{-1}:[\rho \in(a, b) \mapsto \Pi(\rho)] \mapsto$ $[V \in(1 / b, 1 / a) \mapsto-\Pi(1 / V)]$. Оно сводит уравнение в $(32)$ к уравнению

$$
\frac{d^{2}}{d V^{2}} \Phi(V)=\frac{1}{V} \frac{2 \varepsilon \frac{d}{d V} \Phi(V)}{f(V)-\Phi(V)}
$$

с $\frac{d}{d V} \Phi>0$ и $\Phi>f$ на $(1 / b, 1 / a)$, где $\Phi=T^{-1} \Pi$ и $f=T^{-1} p$. Это уравнение отличается от уравнения, рассмотренного в [15], лишш множителем $1 / V$ в правой части. Так как $a>0$, этот множитель непрерьвен и ограничен на $(1 / b, 1 / a)$, и доказательство из [15] проводится без изменений. Получаем сушествование строго возрастающего выпуклого решения $\Phi \in C[1 / b, 1 / a] \cap C^{2}(1 / b, 1 / a)$ задачи (35) такого, что $\Phi(1 / a)=f(1 / a), \Phi(1 / b)=f(1 / b)$. Значит, $\Pi=T \Phi \in C[a, b] \cap C^{2}(a, b)$ и П удовлетворяет (32).

Для $a=0$ сперва найдем для каждого $\delta \in(0, b)$ функцию $\Pi_{\delta} \in C[0, b] \cap C^{2}(\delta, b)$ такую, что

$$
\begin{gathered}
\rho \ddot{\Pi}_{\delta}+2 \dot{\Pi}_{\delta}=\frac{2 \varepsilon \dot{\Pi}_{\delta}}{p-\Pi_{\delta}}, \quad \dot{\Pi}_{\delta}>0, \quad p-\Pi_{\delta}>0 \quad \text { на }[\delta, b), \\
\Pi_{\delta}(b)=p(b),\left.\quad \Pi_{\delta}\right|_{[0, \delta]}=-k \varepsilon .
\end{gathered}
$$

Доказательство существования проводится аналогично. По лемме 2(b) существует функция П на $[0, b]$ такая, что $\Pi_{\delta} \uparrow \Pi$ при $\delta \downarrow 0$. Кроме того, применяя еще раз 
рассуждение из [15] к функциям $T^{-1} \Pi_{\delta}$, находим, что $T^{-1} \Pi \in C^{2}(1 / d, 1 / c)$ и что $T^{-1}$ П удовлетворяет (35) на $(1 / d, 1 / c)$ для любого отрезка $[1 / d, 1 / c] \subset(1 / b,+\infty)$. Поэтому П $\in C^{2}(0, b)$ и вьполняется уравнение (32). Кроме того, как и в [15], непрерывность П в точках 0 и $b$ следует из леммы 2 (a) при помощи сравнения со специальными решениями уравнения (32). Поэтому П $(0)=-k \varepsilon$ и П $(b)=p(b)$ по построению $\Pi_{\delta}$.

ДОКАЗАТЕЛЬСТВО ПРЕДЛОЖЕНИЯ 4 . Пусть $a<b$, а $P(\cdot)$ яВЛяется оболочкой функции $p(\cdot)$ на $[a, b]$. Возьмем $\alpha>0$ и построим барьерную функцию $\Upsilon_{\alpha} \in$ $C^{2}(a, b) \cap C[a, b]$ такую, что $\rho \ddot{\Upsilon}_{\alpha}+2 \dot{\Upsilon}_{\alpha} \geqslant m(\alpha)>0$ и $\alpha / 2 \leqslant P-\Upsilon_{\alpha}<\alpha$ на $(a, b)$. Применим лемму $2\left(\right.$ a) к $\Pi^{\varepsilon}$ и $\Upsilon_{\alpha}$. По лемме $3\left(\right.$ a) $\frac{1}{\rho} \dot{\Pi}^{\varepsilon}$ равномерно ограничена на любом отрезке $[c, d] \subset(a, b)$. Следовательно, $P \geqslant \prod^{\varepsilon} \geqslant \Upsilon_{\alpha}$ на $[a, b]$ для достаточно малых $\varepsilon$.

В заключение отметим следуюшее свойство решений (32).

ЛЕмма 4. Пусть П $(\cdot ; \sigma)$ удовлетворяет (32) с $а$ а $=0, k=-\sigma \in[0,1)$. Тогда $\dot{\Pi}(\rho ; \sigma) \rightarrow 0$ при $\rho \rightarrow 0$ и интеграл $S(\sigma)=\int_{0}^{b} \sqrt{\dot{\Pi}(r ; \sigma)} \frac{d r}{r}$ конечен. В дополнение, $S(\sigma) \uparrow+\infty$ при $\sigma \downarrow-1$.

ДокаЗАТЕЛЬСтво. Докажем, что $S(\sigma)$ сходится (на нижнем пределе). Положим $\varkappa=1-(1-k) / 2 \in[1 / 2,1)$. Так как П, $p$ непрерывны на $[0, b]$ и $p(0)-\Pi(0 ; \sigma)=k \varepsilon$, сушествует $\delta>0$ такое, что $p-\Pi \leqslant \varkappa \varepsilon$ на $(0, \delta)$. Согласно $(32)$ на $(0, \delta)$ имеем

$$
\dot{\Pi}(\delta ; \sigma) \geqslant \dot{\Pi}(\rho ; \sigma)+\int_{\rho}^{\delta}\left(\frac{\varepsilon}{\varkappa \varepsilon}-1\right) \frac{2}{r} \dot{\Pi}(r ; \sigma) d r .
$$

Отсюда $\dot{\Pi}(\rho ; \sigma) \leqslant$ const $\rho^{2(1 / \varkappa-1)}$ по неравенству Гронуолла, так что первые два утверждения леммы очевидны.

Теперь рассмотрим функцию $\dot{\Pi}(\cdot ;-1)$. Если $S(-1)$ расходится, то $\lim _{\sigma \downarrow-1} S(\sigma)$ $=+\infty$ по лемме $2(\mathrm{~b})$ и по теореме Леви. Предположим, что $S(-1)<+\infty$, и придем к противоречию.

Рассмотрим функцию $\Xi_{+}(\rho)=\sqrt{\dot{\Pi}_{+}(\rho ;-1)}-\int_{\rho}^{b} \sqrt{\dot{\Pi}_{+}(r ;-1)} \frac{d r}{r}$. Из (32) находим, что $\dot{\Xi}_{+}>0$ на $(0, b]$. Так как $\Xi_{+}(\rho) \geqslant-S(-1)>-\infty, \Xi_{+}$стремится к конечному пределу при $\rho \rightarrow 0$. Следовательно, предел $\sqrt{\dot{\Pi}_{+}(\rho ;-1)}$ при $\rho \rightarrow 0$ существует и равен нулю. Теперь положим $\xi_{+}=\lim _{\rho \rightarrow 0} \Xi_{+}(\rho)$ и определим функции класса $C^{1} \rho, u: \xi \in\left[\xi_{+},+\infty\right) \mapsto \rho(\xi), u(\xi)$ как в формулах $(24)-(26)$, полагая $u_{+}=0$. Переписывая (32) в переменных $\xi, \rho, u$, получаем $\rho\left(\xi_{+}\right)=0, \xi_{+}-u\left(\xi_{+}\right)=0$, $u^{\prime}\left(\xi_{+}+0\right)=1$ и

$$
\varepsilon\left(u^{\prime}(\xi)-u^{\prime}\left(\xi_{+}+0\right)\right)=-\int_{\xi_{+}}^{\xi}(\zeta-u(\zeta)) \rho(\zeta) u^{\prime}(\zeta) d \zeta+p(\rho(\xi))
$$

для всех $\xi>\xi_{+}$. Как показано в части III) доказательства леммы 1 , эти свойства несовместны. 
Для $\sigma \in(-1,+\infty)$ обозначим через $S_{ \pm}^{\varepsilon}(\sigma)$ интегралы $\int_{(\sigma)+}^{\rho_{ \pm}} \sqrt{\dot{\Pi}_{ \pm}^{\varepsilon}(r ; \sigma)} \frac{d r}{r}$, где $\Pi_{ \pm}^{\varepsilon}(\cdot ; \sigma)$ обозначает единственное решение $(20)-(22)$ согласно предложению 3 . По лемме $4 S_{ \pm}^{\varepsilon}(\sigma)$ конечны при $\sigma \in(-1,0]$; очевидно, то же верно для $\sigma \in(0,+\infty)$.

Следующий шаг состоит в нахождении $\sigma$, удовлетворяющего условию (23), т.е.

$$
u_{+}-u_{-}=S_{+}^{\varepsilon}(\sigma)+S_{-}^{\varepsilon}(\sigma)
$$

как показано вьше, это условие имеет смысл. Докажем, что для фиксированных $\rho_{ \pm}$и $\varepsilon(23)$ устанавливает взаимно однозначное соответствие между $\sigma \in(-1,+\infty)$ и $u_{+}-u_{-} \in \mathbb{R}$ при условии, что $p(\cdot)$ удовлетворяет $(4)$. Для $\rho_{0}=(\sigma)^{+} \in[0,+\infty)$ обозначим через $P_{ \pm}\left(\cdot ; \rho_{0}\right)$ оболочки функции $p(\cdot)$ на $\overline{I\left(\rho_{0}, \rho_{ \pm}\right)}$соответственно. Удобно доопределить $\Pi_{ \pm}^{\varepsilon}(\cdot ; \sigma), P_{ \pm}\left(\cdot ; \rho_{0}\right)$ по непрерывности на $\mathbb{R}^{+}$, полагая их постоянными на каждой из компонент $\mathbb{R}^{+} \backslash I\left(\rho_{0}, \rho_{ \pm}\right)$.

ЛЕмма 5. Во введенных выше обозначениях при $\sigma \in(-1,+\infty), \rho_{0}=(\sigma)^{+}$ верно следующее:

(а) для всех $\rho \in \mathbb{R}^{+}$и $\varepsilon>0$ функиии $\sigma \mapsto \Pi_{ \pm}^{\varepsilon}(\rho ; \sigma)$ не убивают; то жее верно для $\rho_{0} \mapsto P_{ \pm}\left(\rho ; \rho_{0}\right)$;

(b) для всех $\rho \in \mathbb{R}^{+} u \varepsilon>0$ функиии $\sigma \mapsto \operatorname{sign}\left(\rho_{ \pm}-\rho_{0}\right) \dot{\Pi}_{ \pm}^{\varepsilon}(\rho ; \sigma)$ не возрастают; то жее верно для $\rho_{0} \mapsto \operatorname{sign}\left(\rho_{ \pm}-\rho_{0}\right) \dot{P}_{ \pm}\left(\rho ; \rho_{0}\right)$;

(c) для всех $\varepsilon>0$ отображсения $\sigma \mapsto \Pi_{ \pm}^{\varepsilon}(\cdot ; \sigma)$ непрерьвньи в топологии $L^{\infty}\left(\mathbb{R}^{+}\right) ;$то жсе верно для $\rho_{0} \mapsto P_{ \pm}\left(\cdot ; \rho_{0}\right) ;$

(d) для всех $\varepsilon>0$ функиии $\sigma \mapsto S_{ \pm}^{\varepsilon}(\sigma)$ непрерьвниц и строго убьвают; то же верно для функиий $\rho_{0} \mapsto \int_{\rho_{0}}^{\rho_{ \pm}} \sqrt{\dot{P}_{ \pm}\left(r ; \rho_{0}\right)} \frac{d r}{r}$.

ДокАЗАтЕльство. Свойства (a)-(c) для $\varepsilon>0$ следуют из леммы 2. Тогда $(\mathrm{a})-(\mathrm{c})$ для $P_{ \pm}$следуют из предложения 4 и леммы $3(\mathrm{~b})$. Из (b) и теоремы Леви находим $(\mathrm{d})$.

ЗАмЕчАнИЕ 2. Имеет место следующее свойство экстремальности: на верхней оболочке $P(\cdot)$ функции $p(\cdot)$ на $[a, b] \subset(0,+\infty)$ интеграл $\int_{a}^{b} \sqrt{\dot{Q}(r)} \frac{d r}{r}$ достигает наибольшего значения в классе всех $Q(\cdot)$, удовлетворяющих свойствам $(7)\left(\mathrm{i}^{\prime}\right)$, $\left(\right.$ ii $\left.^{\prime \prime}\right)$, таких, что $Q(a)=p(a), Q(b)=p(b)$.

Для доказательства достаточноперейтик $F(\cdot)=T^{-1} P(\cdot)$ и $G(\cdot)=T^{-1} Q(\cdot)$. Имеем $F(1 / a)=G(1 / a), F(1 / b)=G(1 / b)$ и функции $F, G$ выпуклы вниз на $[1 / b, 1 / a]$. Тогда

$$
\begin{aligned}
\int_{a}^{b}(\sqrt{\dot{P}(r)}-\sqrt{\dot{Q}(r)}) \frac{d r}{r} & =\int_{1 / b}^{1 / a}\left(\sqrt{\frac{d}{d V} F(v)}-\sqrt{\frac{d}{d V} G(v)}\right) d v \\
& =\int_{1 / b}^{1 / a} \frac{\frac{d}{d V}(F(v)-G(v))}{\sqrt{\frac{d}{d V} F(v)}+\sqrt{\frac{d}{d V} G(v)}} d v \\
& =-\int_{1 / b}^{1 / a}(F(v)-G(v)) d\left(\frac{1}{\sqrt{\frac{d}{d V} F(v)}+\sqrt{\frac{d}{d V} G(v)}}\right)
\end{aligned}
$$


в смысле интеграла Стилтьеса. Учитьвая возрастание функций $\frac{d}{d V} F(V), \frac{d}{d V} G(V)$ на $[1 / b, 1 / a]$ и определение $P(\cdot)$, находим, что $\int_{a}^{b}(\sqrt{\dot{P}(r)}-\sqrt{\dot{Q}(r)}) \frac{d r}{r} \geqslant 0$.

Лемма 6. Пусть заданы $\rho_{ \pm}, u_{ \pm}$, и пусть выполнено (4). Тогда

(а) для любого $\varepsilon>0$ имеем $S_{ \pm}^{\varepsilon}(\sigma) \rightarrow-\infty$ при $\sigma \rightarrow+\infty$,

(b) имеем $\int_{\rho_{0}}^{\rho_{ \pm}} \sqrt{\dot{P}_{ \pm}\left(r ; \rho_{0}\right)} \frac{d r}{r} \leqslant S_{ \pm}^{\varepsilon}\left(\rho_{0}\right)$ для всех $\rho_{0}>0, \varepsilon>0 ;$ в частнос$m u, \int_{\rho_{0}}^{\rho_{ \pm}} \sqrt{\dot{P}_{ \pm}\left(r ; \rho_{0}\right)} \frac{d r}{r} \rightarrow-\infty$ npu $\rho_{0} \rightarrow+\infty$.

ДокАЗАтЕльство. Предположим, что $S^{+}(\sigma)$ ограничено снизу некоторой постоянной $-M \in \mathbb{R}^{-}$. Положим $V_{0}:=1 /(\sigma)^{+}, V_{+}:=1 / \rho_{+}>V_{0}$ и выполним преобразование $T^{-1} p=f, T^{-1} \Pi_{+}^{\varepsilon}(\cdot ; \sigma)=\Phi\left(\cdot ; V_{0}\right)$. Функция $\Phi\left(\cdot ; V_{0}\right)$ выпукла вниз на $\left[V_{0}, V_{+}\right], \Phi\left(V_{0} ; V_{0}\right)=f\left(V_{0}\right), \Phi\left(V_{+} ; V_{0}\right)=f\left(V_{+}\right)$и $\Phi\left(\cdot ; V_{0}\right)$ удовлетворяет уравнению $(35)$ на $\left(V_{0}, V_{+}\right)$. Отметим, что $f\left(V_{0}\right) \rightarrow-\infty$ при $V_{0} \rightarrow 0$ согласно (4). Поэтому из выпуклости $\Phi\left(\cdot ; V_{0}\right)$ вытекает, что $\Phi\left(V_{+} / 2 ; V_{0}\right) \rightarrow-\infty$ при $V_{0} \rightarrow 0$; с другой стороны,

$$
\frac{V_{+}}{2} \sqrt{\frac{d}{d V} \Phi\left(\frac{V_{+}}{2} ; V_{0}\right)} \leqslant-S_{+}^{\varepsilon}(\sigma) \leqslant M
$$

для всех $V_{0} \in\left(0, V_{+} / 2\right)$. Следовательно, семейство функций $\left\{\Phi\left(\cdot ; V_{0}\right)\right\}_{V_{0} \in\left(0, V_{+} / 2\right)}$ таково, что $\Phi\left(V_{+} / 2 ; V_{0}\right) \downarrow-\infty$ при $V_{0} \downarrow 0, \dot{\Phi}\left(V_{+} / 2 ; V_{0}\right) \leqslant\left(2 M / V_{+}\right)^{2}$ равномерно по $V_{0}, \Phi\left(V_{+} ; V_{0}\right)=f\left(V_{+}\right)$и $\Phi\left(\cdot ; V_{0}\right)$ удовлетворяет $(35)$ на $\left(V_{+} / 2, V_{+}\right)$. Сравним $\Phi\left(\cdot ; V_{0}\right)$ с решением $\Psi(\cdot)$ задачи Коши $\Psi\left(V_{+} / 2\right)=\Phi\left(V_{+} / 2 ; V_{0}\right), \dot{\Psi}\left(V_{+} / 2\right)=$ $\left(2 M / V_{+}\right)^{2}+1$ для (35). Для достаточно малых $V_{0} \Psi(\cdot)$ определена на $\left[V_{+} / 2, V_{+}\right]$ и $\Psi\left(V_{+}\right)<f\left(V_{+}\right)$. Можно применить принцип максимума (лемма $\left.2(\mathrm{~b})\right)$ к уравнению (35). Находим, что $\Phi\left(V_{+} ; V_{0}\right)<\Psi\left(V_{+}\right)<f\left(V_{+}\right)$для достаточно малых $V_{0}$. Это противоречие доказывает (a).

Свойство (b) следует из (a) и из замечания 2.

Из предложений 1-3 и лемм 4-6 легко получаем основной результат этого параграфра:

TеОРема 1. Пусть $p(\cdot)$ непрерывна и строго возрастает на $\mathbb{R}^{+}$и верно (4). Тогда для любих $\rho_{ \pm}>0, u_{ \pm} \in \mathbb{R}, \varepsilon>0$ существует единственное решение задачи (1), (2) в смысле определения 2.

ЗАмЕчанИЕ 3 . Легко видеть, что если $p(\cdot)$ не удовлетворяет условию (4), то, вообще говоря, не существует ограниченного автомодельного решения задачи $(1),(2)$ для произвольных данных $\rho_{ \pm}, u_{ \pm}$. Согласно лемме 6(b) точнып условием на разность $u_{+}-u_{-}$, так что имеет место сушествование решения для положительных $\varepsilon$ в некоторой непустой окрестности нуля, является неравенство

$$
-\left(u_{+}-u_{-}\right)<\lim _{\rho_{0} \rightarrow+\infty} \int_{\rho_{+}}^{\rho_{0}} \sqrt{\dot{P}_{+}\left(r ; \rho_{0}\right)} \frac{d r}{r}+\lim _{\rho_{0} \rightarrow+\infty} \int_{\rho_{-}}^{\rho_{0}} \sqrt{\dot{P}_{-}\left(r ; \rho_{0}\right)} \frac{d r}{r}
$$

где $P_{ \pm}\left(\cdot ; \rho_{0}\right)$ - оболочки $p(\cdot)$ на $\left[\rho_{+}, \rho_{0}\right]$ и $\left[\rho_{-}, \rho_{0}\right]$ соответственно. 


\section{§3. Допустимое решение задачи (3), (2)}

В этом параграффе изучается глобальная (по отношению к данным $\rho_{ \pm}, u_{ \pm}$) разрешимость задачи (3), (2) в классе решений, допустимых по критерию веера волн (см. [9], [11], [3]).

ОПрЕДЕЛЕНИЕ 3 . Пусть $(\rho, u)$ - пара функций таких, что $\rho \in L^{\infty}\left(\mathbb{R}^{+} \times \mathbb{R} ; \mathbb{R}^{+}\right)$ и $u \in L^{\infty}(\{\rho>0\} ; \mathbb{R})$, где $\{\rho>0\}:=\left\{(t, x) \in \mathbb{R}^{+} \times \mathbb{R}: \bar{\rho}(t, x)>0\right\}$, а $\bar{\rho}$ является п.в. определенным представителем $\rho$. Тогда $(\rho, u)$ является решением (3), (2), допустимым по критерию веера волн, если

(i) уравнения $\rho_{t}+q_{x}=0, q_{t}+(e+p(\rho))_{x}=0$ выполнены в $\mathscr{D}^{\prime}\left(\mathbb{R}^{+} \times \mathbb{R}\right)$, где $q=\rho u, e=\rho u^{2}$ на множестве $\{\rho>0\}$ и $q=0, e=0$ на его дополнении;

(ii) имеем ess $\lim _{t \downarrow 0}\left(\|\rho(t, \cdot)-\rho(0, \cdot)\|_{L^{1}(-R, R)}+\|u(t, \cdot)-u(0, \cdot)\|_{L^{1}(-R, R)}\right)=0$ для всех $R>0$, где $\rho(0, \cdot), u(0, \cdot)$ даны в $(2)$;

(iii) в дополнение, сушествует последовательность $\left\{\varepsilon_{n}\right\}_{n \in \mathbb{N}} \subset(0,+\infty), \varepsilon_{n} \rightarrow 0$ при $n \rightarrow \infty$, такая, что $\rho^{\varepsilon_{n}} \rightarrow \rho, \rho^{\varepsilon_{n}} u^{\varepsilon_{n}} \rightarrow q$ и $\rho^{\varepsilon_{n}}\left(u^{\varepsilon_{n}}\right)^{2} \rightarrow e$ п.в. на $\mathbb{R}^{+} \times \mathbb{R}$ при $n \rightarrow \infty$, где $\left(\rho^{\varepsilon_{n}}, u^{\varepsilon_{n}}\right)$ является решением задачи $(1),(2)$ в смысле определения 2 .

Отметим, что согласно п. (i) определения $3 u$ не определена в точках вакуума. Условия (i), (ii) определяют слабое решение, тогда как (iii) представляет собой дополнительный критерий отбора решений, более ограничительньй, чем (i), во всех случаях, когда $p(\cdot)$ не вырождается в $p(\rho)=$ const $/ \rho$ на некотором интервале в $\mathbb{R}^{+}$. В этом случае можно построить бесконечно много решений, не являющихся допустимьми (ср. с [17]).

В силу свойства (iii) допустимое по критерию веера волн решение является автомодельным. Согласно формулам (38)-(43) и теореме 2 (см. ниже) оно единственно и имеет следующую структуру веера волн. Решение содержит не более трех “главных" промежутков постоянства: $\left(\rho_{-}, u_{-}\right)$на $-\infty$; вакуум $\rho \equiv 0$ или состояние $\left(\rho_{0}, u_{0}\right), \rho_{0}>0$, в окрестности единственной точки $\xi_{0}$ такой, что существует $\lim _{\xi \rightarrow \xi_{0}} u(\xi)=\xi_{0}$ (в случае, когда нет вакуума); и $\left(\rho_{+}, u_{+}\right)$на $+\infty$. Эти состояния разделены двумя веерами волн первого и второго семейства соответственно (см. [19]). Каждый веер волн представляет собой последовательность разрывов, волн разрежения, контактных разрывов и (в случае, если $p(\cdot)$ не является гладкой) промежутков постоянства, представляющих собой вырожденные волны разрежения. В решении может быть не более одного промежутка вакуума, и таким промежутком может быть только среднее "главное" состояние. В этом случае $u(\xi)-\xi \rightarrow 0$, когда $\xi$ стремится к граничным точкам состояния вакуума (см. (39), (41) и (46) ниже). Необходимым и достаточным условием появления вакуума является неравенство

$$
u_{+}-u_{-} \geqslant \int_{0}^{\rho_{+}} \sqrt{\dot{P}_{+}(r ; 0)} \frac{d r}{r}+\int_{0}^{\rho_{-}} \sqrt{\dot{P}_{-}(r ; 0)} \frac{d r}{r}
$$

где $P_{ \pm}(\cdot ; 0)$ определены ниже. Все эти свойства могут быть получены из следую- 
щих формул для решения, допустимого по критерию “веера волн” :

$$
\begin{gathered}
\rho(t, x)=\rho(x / t)= \begin{cases}{\left[\Xi_{-}\right]^{-1}(x / t),} & x / t<\xi_{-}, \\
{\left[\Xi_{+}\right]^{-1}(x / t),} & \xi_{+}<x / t,\end{cases} \\
\equiv \begin{cases}{\left[\Xi_{-}\right]^{-1}(x / t),} & x / t<\xi_{-}, \\
\rho_{0}, & \xi_{-}<x / t<\xi_{+}, \\
{\left[\Xi_{+}\right]^{-1}(x / t),} & \xi_{+}<x / t,\end{cases} \\
u(t, x)=u(x / t)= \begin{cases}U_{-} \circ\left[\Xi_{-}\right]^{-1}(x / t), & x / t<\xi_{-}, \\
U_{-}\left(\rho_{0}\right)=U_{+}\left(\rho_{0}\right), & \xi_{-}<x / t<\xi_{+}\left(\text {в случае } \rho_{0}>0\right), \\
U_{+} \circ\left[\Xi_{+}\right]^{-1}(x / t), & \xi_{+}<x / t,\end{cases}
\end{gathered}
$$

где

$$
\begin{aligned}
& U_{ \pm}(\rho):=u_{ \pm} \mp \int_{\rho}^{\rho_{ \pm}} \sqrt{\dot{P}_{ \pm}\left(r ; \rho_{0}\right)} \frac{d r}{r} \quad \text { для } \rho \in \overline{I\left(\rho_{0}, \rho_{ \pm}\right)}, \\
& \Xi_{ \pm}(\rho):=U_{ \pm}(\rho) \pm \sqrt{\dot{P}_{ \pm}\left(\rho ; \rho_{0}\right)} \quad \text { для } \rho \in I\left(\rho_{0}, \rho_{ \pm}\right),
\end{aligned}
$$

$\xi_{ \pm}$определены соотношениями

$$
\begin{aligned}
& \xi_{ \pm}:=\lim _{\rho \in I\left(\rho_{0}, \rho_{ \pm}\right), \rho \rightarrow \rho_{0}} \Xi_{ \pm}(\rho) \text { при } \rho_{0} \neq \rho_{ \pm}, \\
& \xi_{-}:=-\infty \text { и/или } \xi_{+}:=+\infty \text { при } \rho_{0}=\rho_{-} \text {и } / \text { или } \rho_{0}=\rho_{+},
\end{aligned}
$$

$\rho_{0}$ равно нулю, если выполняется (37), и $\rho_{0}$ является единственным значением в интервале $(0,+\infty)$, удовлетворяющим соотношению

$$
u_{+}-u_{-}=\int_{\rho_{0}}^{\rho_{+}} \sqrt{\dot{P}_{+}\left(r ; \rho_{0}\right)} \frac{d r}{r}+\int_{\rho_{0}}^{\rho_{-}} \sqrt{\dot{P}_{-}\left(r ; \rho_{0}\right)} \frac{d r}{r}
$$

в случае, если (37) не выполнено; наконец, $P_{ \pm}\left(\cdot ; \rho_{0}\right)$ являются оболочками графика $p(\cdot)$ на $\overline{I\left(\rho_{0}, \rho_{ \pm}\right)}$соответственно (см. определение 1$)$.

Докажем основной результат настоящей статьи.

ТЕОРема 2. Предположим, что непрерывная строго возрастающая функиия $p(\cdot)$ удовлетворяет (4). Пусть $\rho_{ \pm}>0, u_{ \pm} \in \mathbb{R}$. Тогда решение $\left(\rho^{\varepsilon}, u^{\varepsilon}\right)$ задачи (1), (2) стремится при $\varepsilon \downarrow 0$ (в смысле определения 3(iii)) $\kappa(\rho, u)$, задаваемым формулами (38)-(43). Пара функиий $(\rho, u)$ является единственным решением задачи Римана (3), (2) в смысле определения 3.

ДоказАтельство. Возьмем $\varepsilon>0$. Согласно теореме 1 сушествует единственное решение задачи $(1),(2)$. Обозначим это решение через $\left(\rho^{\varepsilon}, u^{\varepsilon}\right)$. Согласно предложениям 1 и $2\left(\rho^{\varepsilon}, u^{\varepsilon}\right)$ соответствует некоторым $\sigma^{\varepsilon} \in(-1,+\infty)$ и $\Pi_{ \pm}^{\varepsilon}\left(\cdot ; \sigma^{\varepsilon}\right)$ таким, что выполняются $(20)-(23)$ и (24)-(28). Рассмотрим множество $\left\{\left(\sigma^{\varepsilon}\right)^{+}\right\} \subset \mathbb{R}^{+} ;$у него есть предельная точка $\rho_{0}$ в $\overline{\mathbb{R}^{+}}$. Выберем последовательность $\varepsilon_{n} \downarrow 0$ такую, что $\sigma^{\varepsilon_{n}} \rightarrow \sigma^{0}$ при $n \rightarrow \infty$; индекс $n$ будем опускать, так 
как ниже будет показано, что предел не зависит от выбора подпоследовательности. Рассмотрим отдельно случаи $\rho_{0} \in(0,+\infty), \rho_{0}=+\infty$ и $\rho_{0}=0$.

a) $\rho_{0} \in(0,+\infty)$. Как в [15], из предложения 4 и леммы $5(\mathrm{a}),(\mathrm{c})$ следует, что

$$
\Pi_{ \pm}^{\varepsilon}\left(\cdot ; \sigma^{\varepsilon}\right) \rightarrow P_{ \pm}\left(\cdot ; \rho_{0}\right) \text { в } L^{\infty}\left(\mathbb{R}^{+}\right) \text {при } \varepsilon \rightarrow 0
$$

где функции $\Pi_{ \pm}^{\varepsilon}, P_{ \pm}$продолжены на $\mathbb{R}^{+}$, как в лемме 5 . Аналогично, доопределим $U_{ \pm}^{\varepsilon}, U_{\varepsilon}$ в $(26)$ и (40) до непрерывной на $\mathbb{R}^{+}$функции без увеличения вариации. Согласно лемме $3(\mathrm{~d}) U_{ \pm}^{\varepsilon}(\cdot)$ сходятся к $U_{ \pm}(\cdot)$ равномерно на $\overline{I\left(\rho_{0}, \rho_{ \pm}\right)}$соответственно. Так как (23) и (43) можно переписать в виде $U_{-}^{\varepsilon}\left(\left(\sigma^{\varepsilon}\right)^{+}\right)=U_{+}^{\varepsilon}\left(\left(\sigma^{\varepsilon}\right)^{+}\right)$и $U_{-}\left(\rho_{0}\right)=U_{+}\left(\rho_{0}\right)$ соответственно, находим $(43)$. Из леммы 5 следует, что в случае а) $\rho_{0}$ определяется единственным образом по $p(\cdot), \rho_{ \pm}$и $u_{ \pm}$.

Далее, согласно лемме $3(\mathrm{c})$ и $(41),(42)$ имеем $\Xi_{ \pm}\left(\rho_{1}\right) \leqslant \xi_{-} \leqslant U_{-}\left(\rho_{0}\right)=$ $U_{+}\left(\rho_{0}\right) \leqslant \xi_{+} \leqslant \Xi_{+}\left(\rho_{2}\right)$ для всех $\rho_{1} \in \overline{I\left(\rho_{0}, \rho_{-}\right)}, \rho_{2} \in \overline{I\left(\rho_{0}, \rho_{+}\right)}$. Рассмотрим отдельно случаи $\rho_{0}>\rho_{+}, \rho_{0}=\rho_{+}, \rho_{0}<\rho_{+}$; по лемме $3($ е) находим из $(44),(24)$ и $(38)$, что $\rho^{\varepsilon}(\cdot) \rightarrow \rho(\cdot)$ п.в. на $\left(\xi_{-},+\infty\right)$. Аналогично получаем $\rho^{\varepsilon}(\cdot) \rightarrow \rho(\cdot)$ п.в. на $\left(-\infty, \xi_{+}\right)$, так что сходимость имеет место п.в. на $\xi \in \mathbb{R}$. Следовательно, $u^{\varepsilon}(\cdot) \rightarrow u(\cdot)$ п.в. на $\mathbb{R}$.

b) $\rho_{0}=+\infty$. На самом деле, этот случай невозможен. Действительно, для всех $L>0$ имеем

$$
\begin{aligned}
u_{+}-u_{-} & =\int_{\sigma^{\varepsilon}}^{\rho_{+}} \sqrt{\dot{\Pi}_{+}^{\varepsilon}\left(r ; \sigma^{\varepsilon}\right)} \frac{d r}{r}+\int_{\sigma^{\varepsilon}}^{\rho_{-}} \sqrt{\dot{\Pi}_{-}^{\varepsilon}\left(r ; \sigma^{\varepsilon}\right)} \frac{d r}{r} \\
& \leqslant \int_{L}^{\rho_{+}} \sqrt{\dot{\Pi}_{+}^{\varepsilon}(r ; L)} \frac{d r}{r}+\int_{L}^{\rho_{-}} \sqrt{\dot{\Pi}_{-}^{\varepsilon}(r ; L)} \frac{d r}{r}
\end{aligned}
$$

для достаточно малых $\varepsilon$ согласно лемме $5(\mathrm{~d})$. Переходя к пределу при $\varepsilon \rightarrow 0$, получаем

$$
u_{+}-u_{-} \leqslant \int_{L}^{\rho_{+}} \sqrt{\dot{P}_{+}(r ; L)} \frac{d r}{r}+\int_{L}^{\rho_{-}} \sqrt{\dot{P}_{-}(r ; L)} \frac{d r}{r}
$$

согласно предложению 4 и лемме $3(\mathrm{~d})$. По лемме $6(\mathrm{c})$ правая часть стремится к $-\infty$ при $L \rightarrow+\infty$, если выполняется (4). Таким образом, $u_{+}-u_{-}=-\infty$, что невозможно.

c) $\rho_{0}=0$. Как и в случае а), имеем (44). Поэтому $\dot{\Pi}_{ \pm}^{\varepsilon}\left(\cdot ; \sigma^{\varepsilon}\right) \rightarrow \dot{P}_{ \pm}(\cdot ; 0)$ п.в. на $\left(0, \rho_{ \pm}\right)$соответственно по лемме $3(\mathrm{~b})$. По теореме Фату имеем

$$
\begin{aligned}
u_{+}-u_{-} & =\liminf _{\varepsilon \rightarrow 0}\left(\int_{0}^{\rho_{+}} \sqrt{\dot{\Pi}_{+}^{\varepsilon}\left(r ; \sigma^{\varepsilon}\right)} \frac{d r}{r}+\int_{0}^{\rho_{-}} \sqrt{\dot{\Pi}_{-}^{\varepsilon}\left(r ; \sigma^{\varepsilon}\right)} \frac{d r}{r}\right) \\
& \geqslant \int_{0}^{\rho_{+}} \sqrt{\dot{P}_{+}(r ; 0)} \frac{d r}{r}+\int_{0}^{\rho_{-}} \sqrt{\dot{P}_{-}(r ; 0)} \frac{d r}{r} .
\end{aligned}
$$

Отметим, что поскольку случай b) исключен, а в случае а) установлено, что правая часть (45) необходимо превосходит $u_{+}-u_{-}$, предельная точка $\rho_{0}$ единственна и конечна. Докажем, что $\rho^{\varepsilon} \rightarrow \rho, \rho^{\varepsilon} u^{\varepsilon} \rightarrow \rho u$ и $\rho^{\varepsilon}\left(u^{\varepsilon}\right)^{2} \rightarrow \rho u^{2}$ на множестве $\{\xi: \rho(\xi)>0\}$ и что $\rho^{\varepsilon} \rightarrow 0, \rho^{\varepsilon} u^{\varepsilon} \rightarrow 0$ и $\rho^{\varepsilon}\left(u^{\varepsilon}\right)^{2} \rightarrow 0$ на его дополнении. 
Сперва предположим, что (45) выполняется с равенством. Тогда по-прежнему имеем $U_{-}(0)=U_{+}(0)$. Более того,

$$
\dot{P}(\rho ; 0) \rightarrow 0 \text { при } \rho \rightarrow 0,
$$

если имеется состояние вакуума. Действительно, как в доказательстве леммы 3(c), можно показать, что $\Xi_{ \pm}(\rho)=u_{ \pm} \mp \int_{\rho}^{\rho_{ \pm}} \sqrt{\dot{P}_{ \pm}(r ; 0)} \frac{d r}{r} \pm \sqrt{\dot{P}_{ \pm}(\rho ; 0)}$ монотонны на $\left(0, \rho_{ \pm}\right) ;$с другой стороны, $\int_{\rho}^{\rho_{ \pm}} \sqrt{\dot{P}_{ \pm}(r ; 0)} \frac{d r}{r}$ также монотонны и сходятся при $\rho \downarrow 0$. Поэтому существуют пределы при $\rho \downarrow 0$ функций $\sqrt{\dot{P}_{ \pm}(\rho ; 0)}$ и они равны нулю. Таким образом, $\Xi_{-}\left(\rho_{1}\right) \leqslant \xi_{-}=U_{-}(0)=U_{+}(0)=\xi_{+} \leqslant \Xi_{+}\left(\rho_{2}\right)$ для всех $\rho_{1} \in\left[0, \rho_{-}\right], \rho_{2} \in\left[0, \rho_{+}\right]$; более того, $\rho(\xi)>0$ для всех $\xi \neq \xi_{-}=\xi_{+}$. Сходимость $\rho^{\varepsilon}, u^{\varepsilon}$ к $\rho, u$ соответственно п.в. на $\mathbb{R}$ доказьвается как в случае а).

Теперь предположим, что в (45) имеет место строгое неравенство. Согласно (46) имеем $\Xi_{-}\left(\rho_{1}\right) \leqslant \xi_{-}=U_{-}(0)<U_{+}(0)=\xi_{+} \leqslant \Xi_{+}\left(\rho_{2}\right)$, где $\rho_{1}, \rho_{2}$ введены выше. Схожим образом получаем сходимость $\rho^{\varepsilon}, u^{\varepsilon}$ к $\rho, u$ соответственно п.в. на $\left(-\infty, \xi_{-}\right) \cup\left(\xi_{+},+\infty\right)$. Отметим, что $\Xi_{ \pm}(\rho)$ строго монотонны в точке $\rho=0$, так как $P_{ \pm}(\cdot ; 0)$ строго возрастают и $\dot{P}_{ \pm}(+0 ; 0)=0$. Следовательно, $\rho(\xi) \rightarrow 0$ при $\xi \uparrow \xi_{-}$ и при $\xi \downarrow \xi_{+}$. Для достаточно малого $\varepsilon \rho^{\varepsilon}$ не имеет точек максимума на $\mathbb{R}$, так что для всех $\delta>0$ находим $\max _{\xi \in\left[\xi_{-}-\delta, \xi_{+}+\delta\right]} \rho^{\varepsilon}(\xi)=\max \left\{\rho^{\varepsilon}\left(\xi_{-}-\delta\right), \rho^{\varepsilon}\left(\xi_{+}+\delta\right)\right\}$. Следовательно, $\rho^{\varepsilon} \rightarrow 0$ равномерно на $\left[\xi_{-}, \xi_{+}\right]$. Кроме того, $u^{\varepsilon}$ являются неубывающими на $\mathbb{R}$ функциями для достаточно малых $\varepsilon$, следовательно, они равномерно ограничены; значит, $\rho^{\varepsilon} u^{\varepsilon} \rightarrow 0$ и $\rho^{\varepsilon}\left(u^{\varepsilon}\right)^{2} \rightarrow 0$ на $\left[\xi_{-}, \xi_{+}\right]$.

Таким образом, показано, что $(\rho, u)(\cdot, \cdot)$ удовлетворяет свойствам (i), (iii) определения 3. Кроме того, $(\rho, u)( \pm \infty)=\left(\rho_{ \pm}, u_{ \pm}\right)$согласно $(38)-(40)$. Так как $\rho(\cdot)$, $u(\cdot)$ монотонны на $\pm \infty$, свойство (ii) определения 3 также выполнено.

\section{Список литературы}

1. Kim Y.-J. A self-similar viscosity approach for the Riemann problem in isentropic gas dynamics and the structure of solutions // Preprint, Univ. Madison, 1999.

2. Tzavaras A.E. Elastic as limit of viscoelastic response, in a context of self-similar viscous limits // J. Differential Equations. 1995. V. 123. № 1. P. 305-341.

3. Tzavaras A.E. Wave interactions and variation estimates for self-similar zero-viscosity limits in systems of conservation laws // Arch. Ration. Mech. Anal. 1996. V. 135. P. 1-60.

4. Калашников А.C. Построение обобщенных решений квазилинейных уравнений первого порядка без условий выпуклости как пределов решений параболических уравнений $\mathrm{c}$ малым параметром // Докл. АН СССР. 1959. Т. 127. №1. С. 27-30.

5. Тупчиев В. А. Распад произвольного разрыва для системы двух квазилинейных уравнений первого порядка // ЖВМиМФ. 1964. Т. 4. С. 817-825.

6. Тупчиев В. А. Задача о распаде произвольного разрыва для системы квазилинейных уравнений без условий выпуклости // ЖВМиМФ. 1966. Т. 6. С. 527-547.

7. Тупчиев В. А. О методе введения вязкости при изучении задач с распадом разрыва // Докл. АН ССCР. 1973. Т. 211. С. 55-58.

8. Dafermos C.M. Solutions of the Riemann problem for a class of hyperbolic systems of conservation laws by the viscosity method // Arch. Ration. Mech. Anal. 1973. V. 52. №1. P. 1-9.

9. Dafermos C. M. Structure of solutions of the Riemann problem for hyperbolic systems of conservation laws // Arch. Ration. Mech. Anal. 1974. V. 53. №3. P. 203-217. 
10. Dafermos C.M., DiPerna R. The Riemann problem for certain classes of hyperbolic systems of conservation laws // J. Differential Equations. 1976. V. 20. P. 90-114.

11. Dafermos C.M. Admissible wave fans in nonlinear hyperbolic systems // Arch. Ration. Mech. Anal. 1989. V. 106. P. 243-260.

12. Tzavaras A. E. Viscosity and relaxation approximations for hyperbolic systems of conservation laws // An introduction to recent developments in theory and numerics of conservation laws / ed. D. Kroener et al. Berlin: Springer-Verlag, 1999. P. 73-122.

13. Slemrod M., Tzavaras A.E. A limiting viscosity approach for the Riemann problem in isentropic gas dynamics // Indiana Univ. Math. J. 1989. V. 38. P. 1047-1074.

14. Fan H. T. The structure of solutions of gas dynamic equations and the formation of the vaccuum state // Quart. Appl. Math. 1991. V. 49. P. 29-48.

15. Andreianov $B$. The Riemann problem for $p$-systems with continuous flux function // Ann. Fac. Sci.Toulouse Math. (6). 1999. V. 8. № 3. P. 353-367.

16. Leibovich $L$. Solutions of the Riemann problem for hyperbolic systems of quasilinear equations without convexity conditions // J. Math. Anal. Appl. 1974. V. 45. № 3. P. 81-90.

17. Krejčı P., Straškraba I. A uniqueness criterion for the Riemann problem // Hiroshima Math. J. 1997. V. 27. № 2. P. 307-346.

18. Hoff D., Smoller J. Non-formation of vacuum states for compressible Navier-Stokes equations // Comm. Math. Phys. 2001. V. 216. № 2. P. 255-276.

19. Lax P. D. Hyperbolic systems of conservation laws II // Comm. Pure Appl. Math. 1957. V. 10. № 4. P. 537-566.

Университет Франш-Комте,

Поступила в редакцию

Безансон, Франция

22.11 .2001 и 09.10 .2002 